\title{
Gamma and electron dose response of the electrical conductivity of polyaniline based polymer composites
}

\author{
U.A. Sevil $^{\mathrm{a}}$, O. Güven ${ }^{\mathrm{a}}$, A. Kovács ${ }^{\mathrm{b}, *}$, I. Slezsák $^{\mathrm{c}}$ \\ a Ankara Nuclear Research and Training Center, Besevler, 06100 Ankara, Turkey \\ ${ }^{\mathrm{b}}$ Chemical Research Center, Institute for Isotopes and Surface Chemistry, Hungarian Academy of Sciences, P.O. B. 77, \\ H-1525 Budapest, Hungary \\ ${ }^{\mathrm{c}}$ Zoltán Bay Institute, Budapest, Hungary
}

\begin{abstract}
Conducting polymers, also known as "synthetic metals", have been the subject of wide-spread investigations over the past decade due to their very promising characteristics. It was published earlier that the electrical conductivity of polyaniline (PANI) based polymer composites increases to a significant extent when irradiated to gamma, electron or UV radiation. The aim of the present study was to determine the dose response of PANI samples blended with poly(vinyl chloride) (PVC) and chlorinated poly(propylene) ( $\mathrm{PPCl}$ ) by measuring the high frequency conductance of these blends irradiated to different doses. In order to find the most suitable composition of these blends the concentration of both the PPCl and PVC, and the PANI base were changed, respectively.

These samples were then gamma irradiated and the induced electrical conductivity was measured in the frequency range of $1 \mathrm{kHz}-1 \mathrm{MHz}$ in order to find the most sensitive evaluation conditions. After selecting both, the most suitable measuring conditions as well as the blend compositions, the dose response of the samples was determined in the dose range of 10-250 kGy. With respect to potential dosimetry application the effects of electron irradiation, irradiation temperature and the stability of the irradiated samples have also been investigated.
\end{abstract}

(C) 2003 Elsevier Science Ltd. All rights reserved.

Keywords: Polyaniline composites; Radiation-induced conductance; Radiation dose response

\section{Introduction}

In the past decade intensive investigations have been carried out in connection with the intrinsically conducting polymers also called as "synthetic metals" due to their special phenomena of combining the physical and chemical characteristics of plastics and the electrical properties of metals. Polyaniline (PANI) has been in focus in this respect for many years because of its unique nature since its electrical characteristics can be changed both by changing the oxidation state of the main chain and by protonation of the imine nitrogen atoms (Chiang and MacDiarmid, 1986).

\footnotetext{
*Corresponding author. Fax: + 36-361-392-2548.

E-mail address: akovacs@alpha0.iki.kfki.hu (A. Kovács).
}

PANI, which can be synthesized by oxidizing aniline either electrochemically or chemically, can exist in various oxidation states, which can be characterized by the ratio of amine to imine nitrogen atom (Huang et al., 1986). PANI can be doped either by protonation with a protonic acid or by charge transfer with an oxidation agent. Its electronic and optical properties can possibly be controlled reversibly by varying the doping level. PANI has got excellent environmental and thermal stability in the conducting form, but on the other hand it has inferior chemical properties like insolubility and difficulty in processibility. In order to overcome these unfavourable characteristics and to improve and widen its application possibilities of their use in blends and composites has been extensively studied. Poly(vinyl)chloride (PVC), vinyl chloride/vinyl acetate (PVCco-PvAc) and chlorinated poly(propylene) ( $\mathrm{PPCl}$ ) were 
suggested, tried and some blends even characterized for certain applications under various irradiation conditions (Bodugöz et al., 1998).

Since, e.g., PVC or PPCl undergo a high degree of side chain degradation (resulting mainly loss of $\mathrm{HCl}$ ) when exposed to ionizing radiation these radiolytic products can be captured by the neighbouring PANI molecules thus enhancing the electrical conductivity of the blend. Bodugöz et al. (1998) introduced the radiation induced doping of PVC/PANI, PPCl/PANI and $\mathrm{VCl}$ or $\mathrm{VAc} /$ PANI blends and found increased electrical conductivity with increasing dose.

Among the various applications of PANI (microelectronics, optical displays, antistatic coatings, etc.), it could possibly play an important role in radiation process control. Radiation processing has got increasing importance nowadays in various fields (sterilization, polymer processing, food irradiation, environmental protection, etc.), thus the role of process control to ensure the safe and reliable execution of these technologies is of basic significance. The increasing demands towards the reliable performance of different dosimetry systems on the one hand, while the introduction of new radiation processing technologies, on the other hand require the investigation and introduction of new, reliable, easy-to-use and cheap dosimeters. Due to the standardization of these technologies dose measurements - especially in high dose range - are required now in such cases too, where earlier it was not necessary. Film systems are of particular interest due to the increasing use of electron accelerators. PANI based blends could play important role as thin dosimeter films due to the radiation induced conductivity change, which requires a quick and simple evaluation method. Other advantageous characteristics could be the capability of measuring high doses and the resistivity against humidity and light, which have significant effect on the radiochromic dosimeter films in regular use.

\section{Experimental}

\subsection{Preparation of the films}

In order to find the most suitable film compositions, various types of the PPCl/PANI and PVC/PANI films were prepared by the solution casting method (thickness: $0.20 \pm 0.02 \mathrm{~mm}$ ). The blend films contained $0.5,1.0$ and $1.5 \mathrm{~g} \mathrm{PPCl}$ or PVC, while the PANI base concentrations were 5-10-15-20 wt $\%$ at each preparation. Blends of PANI with PVC were prepared by using tetrahydrofuran solvent, while in the case of PANI/PPCl the solvent was xylene. PANI was prepared chemically by polymerizing the freshly distilled monomer in $1 \mathrm{~mol} / \mathrm{dm}^{3}$ acidic solution using $\left(\mathrm{NH}_{4}\right)_{2} \mathrm{~S}_{2} \mathrm{O}_{8}$ as the oxidizer/ initiator.

\subsection{Irradiation of the film samples}

The PANI/PPCl and the PANI/PVC films were irradiated with gamma and electron radiation. The gamma irradiations were carried out with the pilot scale ${ }^{60} \mathrm{Co}$ gamma irradiation facility of the Institute of Isotopes Ltd. Co. (SSL-01 type, 3 PBq nominal activity). The irradiations were performed at positions of different dose rate $(15$ and $1 \mathrm{kGy} / \mathrm{h})$ within and outside of the source cage. The films were irradiated in a polystyrene holder. The dose rate was measured with the ethanolmonochlorobenzene dosimeter solution. Temperature controlled irradiations were performed at the same irradiation positions by placing the film samples onto a heated surface of a device of controlled heating/cooling. The irradiation temperature in this case was $25^{\circ} \mathrm{C}$ and $50^{\circ} \mathrm{C}$, respectively.

The electron irradiation of the films was carried out with the TESLA type $4 \mathrm{MeV}$ linear electron accelerator of the Institute for Isotope and Surface Chemistry. The film samples were placed below the vertical accelerator tube and were irradiated in pulsed mode. The pulse duration was $2.6 \mu$ s, while the pulse repetition frequency was $50 \mathrm{~Hz}$. The dose rate value, as measured with the ethanol-monochlorobenzene dosimeter solution, was $14.2 \mathrm{kGy} / \mathrm{min}$.

\subsection{Resistance measurement of the film samples}

The gamma and electron irradiated film samples were evaluated after irradiation by measuring their resistance (as the real part of the complex impedance). It was measured by high frequency impedance method, applying $1 \mathrm{MHz}$ measuring frequency by using a capacitive cell. It is a planar capacity consisting of two parallel cylindrical electrodes of 3 and $6 \mathrm{~mm}$ diameter, respectively, in order to decrease the scattered capacity.

\section{Results and discussion}

\subsection{Gamma and electron irradiation of the film samples}

When PPCl/PANI or PVC/PANI films are exposed to ionizing radiation the following process is supposed to happen (Fig. 1).

In order to select the most promising composition of the film samples prepared, 12 different composition of $\mathrm{PPCl} / \mathrm{PANI}$ and PVC/PANI films were gamma irradiated to $150 \mathrm{kGy}$. The resistance and the capacitance of these films were then measured and compared to that of the unirradiated film samples. The capacitance of the irradiated films did not change significantly due to irradiation using our measuring set-up, but the change of resistance was remarkable. 


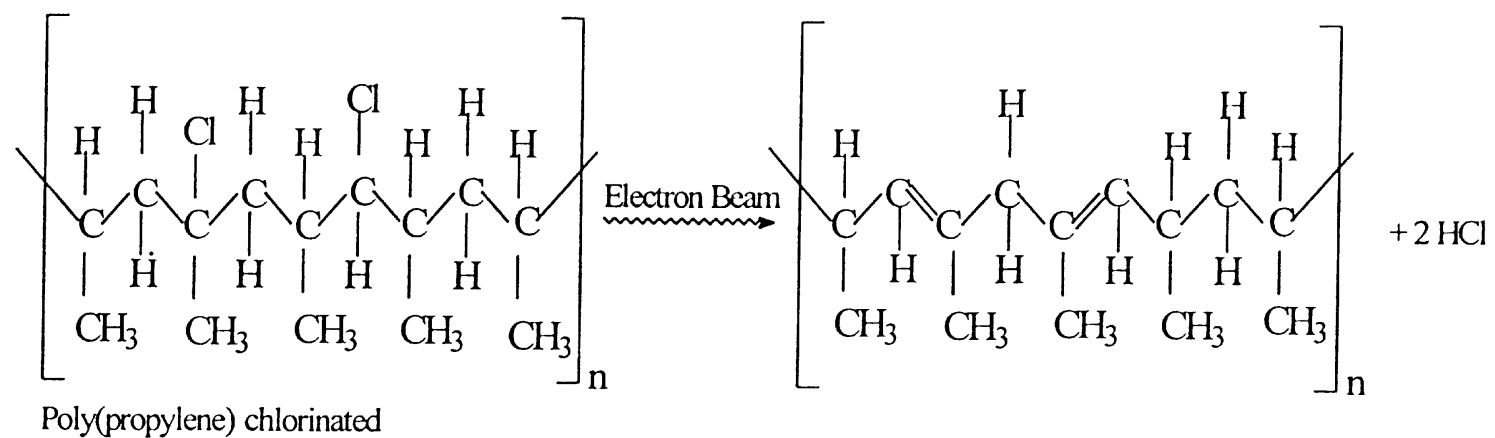

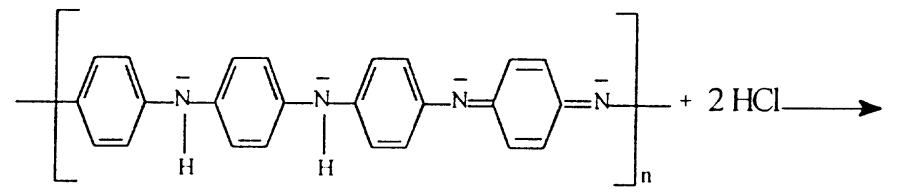

Emeraldine Base

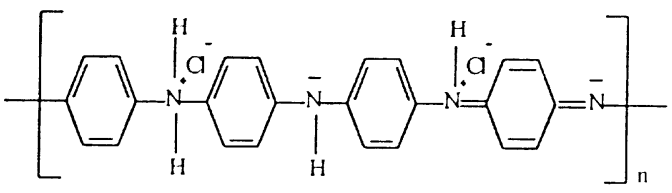

Emeraldine Salt

Fig. 1. Chemical structure of $\mathrm{PPCl}$ and its reaction with PANI base upon gamma/electron irradiation.

In the case of the PPCl-PANI films the biggest change was found in the case of the films containing $15 \%$ and $20 \%$ PANI base at each PPCl concentration. In the case of the sample containing $1.5 \mathrm{~g} \mathrm{PPCl}$ even the film composition containing 10\% PANI base showed reasonable change. Thus, the films containing the optimum concentrations of $\mathrm{PPCl}$ and PANI were selected for further investigations.

In the case of the PVC/PANI films similar trend, but much smaller difference as compared to the $\mathrm{PPCl}$ films was found. Thus only one composition, i.e. the film containing $1.5 \mathrm{~g} \mathrm{PVC}$ and $20 \%$ PANI base was selected for further tests.

These tests involved the gamma irradiation of the selected samples in the dose range of 5-150 kGy in the case of the PPCl films, while in the case of the PVC films this dose range was $5-250 \mathrm{kGy}$ due to the smaller change observed at relatively lower doses.

\subsubsection{Gamma irradiation of PPCl/PANI blends}

Six types of PPCl/PANI blends of different composition were irradiated with gamma radiation in the dose range of $5-150 \mathrm{kGy}$. The resistance of the irradiated films decreased in each case, i.e. the conductance of the films increased with increasing dose in the case of each type of film. The most significant radiation induced change in resistance was observed in the case of the films containing $1.5 \mathrm{~g} \mathrm{PPCl}-15 \mathrm{wt} \%$ PANI (Fig. 2).

\subsubsection{Gamma irradiation of PVCIPANI blends}

In this case, two blends of different composition were investigated by irradiating those with gamma radiation in the dose range of $5-250 \mathrm{kGy}$. Similar behaviour as found in the case of the $\mathrm{PPCl}$ blends with respect to the change of conductance was observed, although the radiation induced relative change was not so significant as shown in Fig. 3 for the blend containing $1.5 \mathrm{~g} \mathrm{PVC}$ and $20 \mathrm{wt} \%$ PANI.

\subsubsection{Electron irradiation of the PANI blends}

The previously selected PPCl/PANI and PVC/PANI blends were electron irradiated too in the dose range of $5-100 \mathrm{kGy}$. The change of resistance/conductance was similar as observed with gamma irradiation, although its extent was relatively smaller with electron irradiation (Fig. 4).

\subsection{Effect of dose rate on the PANI blends investigated}

The effect of dose rate on the PANI blends was investigated by irradiating them both with gamma radiation (using 1 and $15 \mathrm{kGy} / \mathrm{h}$ ) and electron radiation $(14.2 \mathrm{kGy} / \mathrm{min})$. No significant difference was observed between the measured resistance values (Fig. 4), when the PPCl/PANI and PVC/PANI blends were irradiated with gamma radiation using two different dose rates. The much higher dose rate during electron irradiation, on the other hand, resulted in smaller change in resistance with respect to the gamma radiation effect in each type of blends (Fig. 4).

\subsection{Stability of PANI blends after irradiation}

The short-term stability of both types of PANI blends after irradiation - stored under regular laboratory conditions - was studied by measuring the resistance of the gamma and electron irradiated films at different times after irradiation. In the case of each type and 


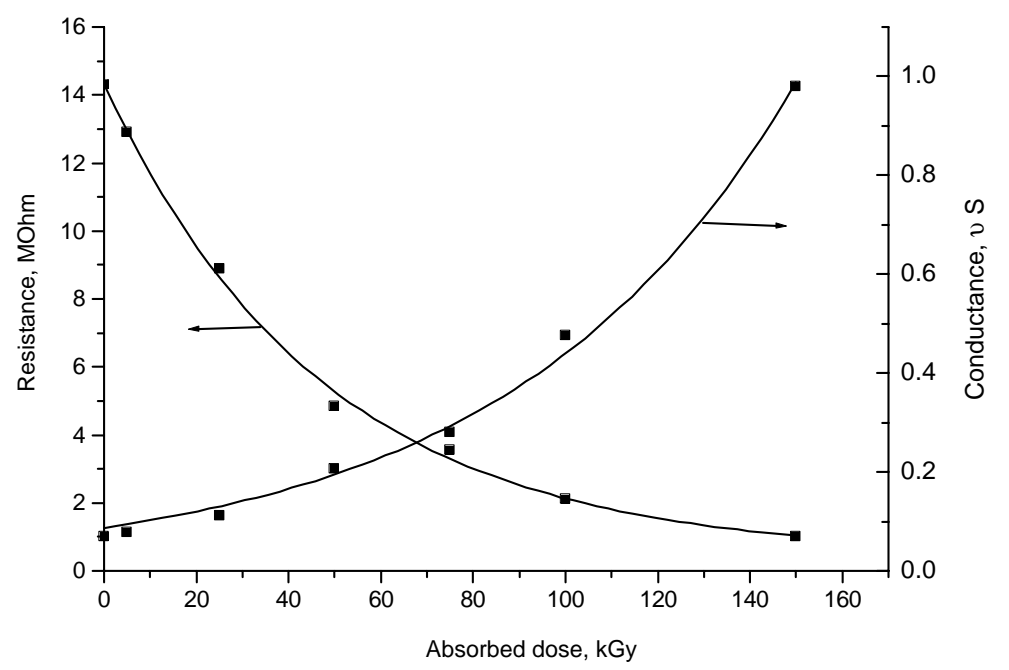

Fig. 2. Effect of dose on the resistance and conductance of the $\mathrm{PPCl} / 15 \%$ PANI blend.

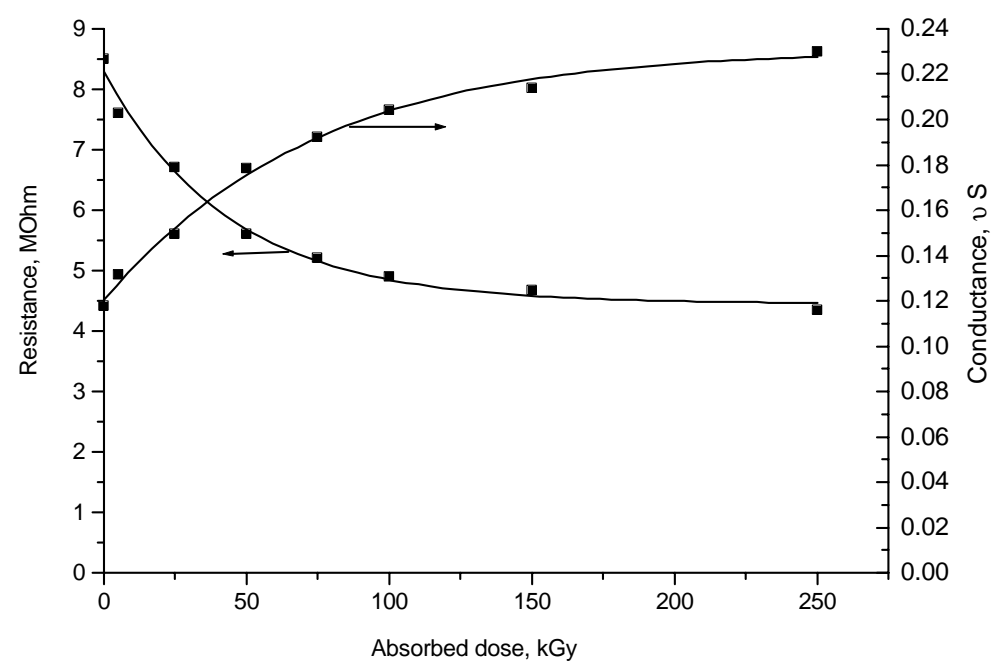

Fig. 3. Effect of dose on the resistance and conductance of the PVC/20\% PANI blend.

composition of blends a slight change (approximately $0.5 \% /$ day) in the measured resistance values was found within a week after irradiation (Fig. 5).

\subsection{Effect of irradiation temperature on the PANI blends}

Preliminary tests to study the effect of irradiation temperature on the radiation induced resistance of the selected PANI blends were carried out by irradiating these blends both at $25^{\circ} \mathrm{C}$ and $50^{\circ} \mathrm{C}$ with gamma irradiation. Comparing these results to each other, it was observed that the resistance of the blends irradiated at elevated temperature increased from $10 \%$ to about $130 \%$ with respect to irradiation at room temperature. Further detailed investigations are on the way to determine the effect of irradiation temperature on the performance of these blends.

\section{Conclusions}

1. The gamma and electron irradiation of the PPCl/ PANI and PVC/PANI blends resulted in significant decrease of the resistance of the blends investigated. That also means that the conductance of these blends increased with increasing dose in the dose range of 5-150 kGy.

2. No significant dose rate effect was observed in the case of irradiating the blend samples with two different dose rates using gamma radiation. When 


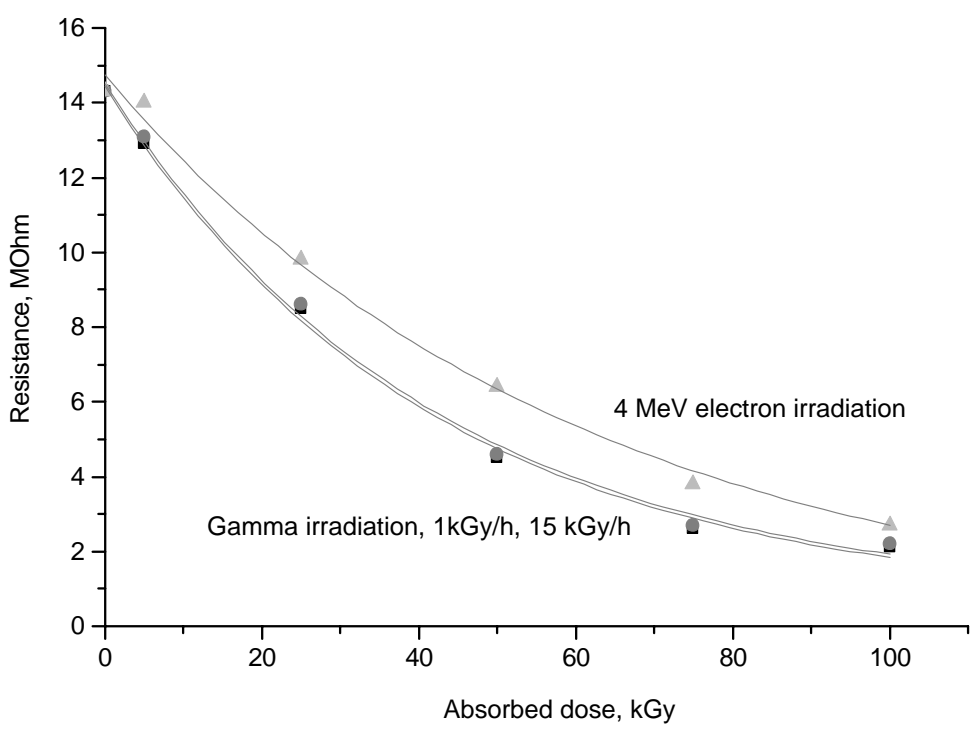

Fig. 4. Effect of dose rate on the gamma and electron irradiated $\mathrm{PPCl} / \mathrm{PANI}$ blends.

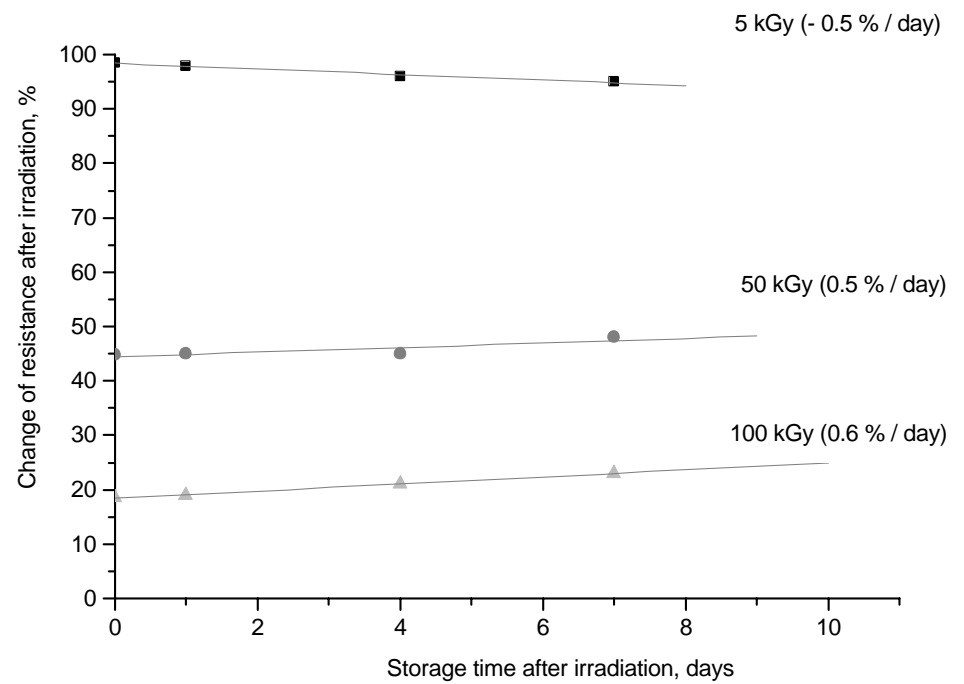

Fig. 5. Effect of short-term storage time on the stability of the irradiated PPCl/PANI blend.

exposing the blends to electron irradiation, however, smaller change in resistance as compared to gamma irradiation was found.

3. According to our preliminary investigations the short-term stability of the blends after irradiation seems to be satisfactory with respect to potential future applications.

4. The increase of irradiation temperature from room temperature to $50^{\circ} \mathrm{C}$ results in the increase of the resistance of these types of blends, but further detailed investigations are needed for their characterization.
5. Based on all these observations the potential dosimetric application of the PANI based blends is possible by using high frequency impedance measurements. According to our investigations the most promising composition of these blends contains $15 \%$ or $20 \%$ PANI base.

It is of basic importance, however, that the blends of the most suitable composition as selected on the basis of these investigations need further detailed characterization and testing at industrial scale irradiation facilities. 


\section{References}

Bodugöz, H., Sevil, U.A., Güven, O., 1998. Radiation-induced conductance in the blends of poly(aniline-base) with poly(vinyl chloride)-co(vinyl acetate). Macromol. Symp. 169, 289-295.
Chiang, J.C., MacDiarmid, A.G., 1986. Polyaniline protonic acid doping of the emeraldine form to the metallic regime. Synth. Met. 13, 193-197.

Huang, W.S., Humphrey, B.D., MacDiarmid, A.G., 1986. Polyaniline: doping structure and derivatives. J. Chem. Soc. Faraday Trans. 82, 2385-2390. 